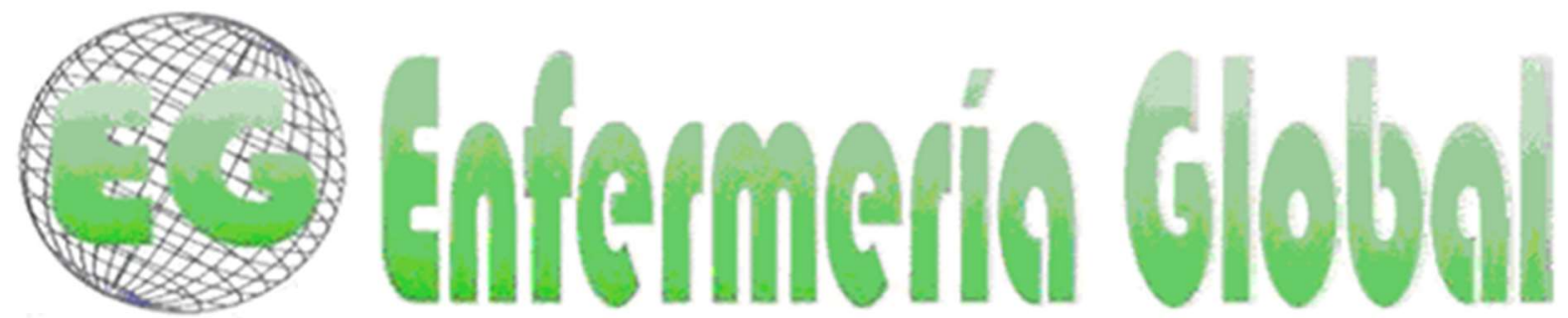

ISSN 1695-6141

Revista electrónica trimestral de Enfermería

$\mathbf{N}^{\circ} 62$

www.um.es/egloball

Abril 2021

\title{
ORIGINALES
}

\section{Estudio preliminar del proceso fin de vida mediante triangulación de datos en un hospital comarcal}

Preliminary study of the end-of-life process through data triangulation in a regional Hospital

María del Mar Alfaya-Góngora ${ }^{1}$

María Angustias Sánchez-Ojeda ${ }^{1}$

Miguel Ángel Gallardo-Vigil²

Silvia Navarro-Prado ${ }^{1}$

${ }^{1}$ Facultad de Ciencias de la Salud de Melilla. Universidad de Granada. Departamento de Enfermería. España.maso@ugr.es

2 Facultad de Educación y del Deporte de Melilla. Universidad de Granada. Departamento de Métodos de Investigación y Diagnóstico en Educación. España.

https://doi.org/10.6018/eglobal.428511

Recibido: $19 / 05 / 2020$

Aceptado: $21 / 12 / 2020$

\section{RESUMEN:}

Objetivo: Conocer las características del proceso de final de vida en un Hospital Comarcal, según los actores implicados, estableciendo los elementos de convergencia/divergencia en el proceso asistencial, toma de decisiones, información y conocimientos.

Método: Estudio observacional, transversal, descriptivo, convergente y analítico de métodos mixtos y triangulación de datos. Se realizó sobre una muestra de 63 sujetos, de los que 25 fueron profesionales, 19 familiares cuidadores y 19 historias clínicas de pacientes fallecidos a lo largo de un año.

Resultados: La triangulación de datos muestra discrepancias entre familiares y profesionales de salud en cuanto a la calidad de la asistencia y la información facilitada. Destaca la falta de registro en las historias clínicas revisadas sobre aspectos del proceso asistencial relacionados con la toma de decisiones o la sedación terminal.

Conclusión: Este estudio proporciona una visión integrada sobre la atención sanitaria en el proceso de final de vida prestada en un Hospital Comarcal. Muestra áreas prioritarias de intervención, para mejorar la calidad de vida en este proceso, como son la implicación del paciente en la toma de decisiones, la cumplimentación adecuada en las historias clínicas y la formación de los profesionales.

Palabras clave: Cuidados Paliativos; Técnicas de Investigación; Atención al paciente; Familia; Personal de Salud.

\section{ABSTRACT:}

Objective: To know the characteristics of the end-of-life process in a Regional Hospital, according to the actors involved, establishing convergence/divergence elements in care process, decision-making, information and knowledge.

Method: Observational, cross-sectional, descriptive, convergent of mixed methods and data 
triangulation study. Carried out on a sample of 63 subjects, of which 25 were health professionals, 19 family caregivers and 19 medical records of patients who died in the course of a year.

Results: Data triangulation shows discrepancies between family members and health professionals regarding care quality and the information provided. The lack of documentation in medical records of care aspects related to decision-making or terminal sedation stands out.

Conclusion: This study provides an integrated view of end-of-life care provided in a Regional Hospital, identifying priority areas of intervention in order to improve quality of life in this process, such as patient involvement in decision-making, appropriate completion of medical records and health professional training.

Key words: Palliative Care; Research Techniques; Patient Care; Family; Health Personnel.

\section{INTRODUCCIÓN}

Los pacientes en situación terminal, así como sus familiares y profesionales de salud que los atienden, se enfrentan a una situación compleja ${ }^{(1)}$ que requiere una atención integral dirigida a cada uno de los actores implicados en este proceso. Los pacientes, además de presentar signos y síntomas físicos como son, entre otros el dolor, desnutrición y los efectos derivados de los tratamentos, requieren atención a otras necesidades como son el respeto a sus decisiones, su intimidad, información adecuada, comprensión, compasión y ayuda para afrontar su situación. Esto implica la necesidad de brindar apoyo psicológico, social y espiritual tanto al paciente como a sus familiares y contar con una formación especializada por parte del equipo de salud que los atiende, especialmente en aspectos bioéticos, habilidades comunicación, apoyo emocional y dinámicas de trabajo en equipo de forma coordinada y efectiva entre los distintos escenarios de la red de asistencia sanitaria(2). La multidisciplinaridad de los equipos nos obliga a que, mas allá de la formación específica y competencias de cada sector profesional, se reciba una formación compartida, en la que cada miembro del equipo conozca que hace el otro y poder ofrecer unos cuidados integrales reales y de calidad.

Sobre los aspectos que determinan una adecuada asistencia en este proceso, la mayoría de los trabajos revisados destacan el acceso a los cuidados paliativos, la formación y experiencia de los profesionales de salud, los objetivos del tratamiento, la prestación de cuidados integrales, la participación de pacientes y familiares, la planificación del proceso de final de vida y el respeto de los deseos del paciente ${ }^{(3-5)}$.

En la práctica, la alta tasa de hospitalización de pacientes que se encuentran en esta situación se asocia con tratamientos agresivos dirigidos más a prolongar la vida que a aliviar síntomas. Asimismo, durante la hospitalización es frecuente la escasa información, comunicación y participación del paciente en su proceso ${ }^{(5,6)}$.

La existencia de distintos modelos organizativos, heterogeneidad de recursos, diversidad del nivel de formación de los profesionales de salud, así como los aspectos culturales, políticos, éticos y religiosos, condicionan el abordaje del proceso de final de vida. Todos estos factores configuran una complejidad asistencial que debe responder a diferentes áreas de necesidades conociendo la perspectiva de los diferentes actores implicados en el proceso ${ }^{(7-10)}$.

Diversos estudios han analizado estas cuestiones desde la experiencia de los profesionales de salud, destacando la necesidad de una formación adecuada para ofrecer una atención de calidad(11-13). Referente a la elección del lugar en el que se 
desarrollan los cuidados, algunos autores encuentran discrepancias en la percepción que profesionales y familiares tienen, al decidir optar por atención en el domicilio, destacando la importancia de aprender a trabajar conjuntamente con una buena coordinación profesional-cuidador, la dotación de recursos y la participación del paciente en la toma de esta decisión ${ }^{(6)}$. Es importante analizar la calidad de vida de los cuidadores, el grado de conocimiento referente al diagnóstico y pronóstico, la comunicación entre pacientes, familiares y profesionales, en unidades hospitalarias que se presten cuidados fin de vida(14).

Este trabajo se desarrolla en el Hospital Comarcal de Melilla, único en la ciudad y con una capacidad de 172 camas, que atiende a una población de 86.120 habitantes, según los datos registrados del Instituto Nacional de Estadística de $2017^{(15)}$. El carácter fronterizo de esta ciudad hace que este centro sanitario atienda a una población con marcada diversidad cultural que incrementa la complejidad de la atención sanitaria en el proceso de final de vida. Los objetivos planteados en este estudio fueron: analizar cómo se desarrollan los procesos de atención de final de vida en pacientes oncológicos y no oncológicos hospitalizados en esta ciudad según los actores implicados, identificando elementos de convergencia/divergencia en categoría preestablecidas: proceso asistencial, información y conocimiento sobre diagnostico, pronóstico, tratamiento y toma de decisiones.

\section{MÉTODO}

\section{Diseño}

El marco metodológico fue el de la investigación mediante métodos mixtos, orientación que permite una investigación integral para abordar situaciones complejas, bajo un enfoque que combina dos o más estrategias, cuantitativas y/o cualitativas, dentro de un único proyecto de investigación(16).

Se han utilizado distintas fuentes y métodos de recogida de datos para buscar la convergencia/divergencia mediante la triangulación ${ }^{(16,17)}$. En este caso, la muestra está constituida por tres categorías de participantes (familiares, pacientes y profesionales) y diferentes perspectivas, sobre las que se realizó un estudio de diseño observacional, transversal, descriptivo, convergente y analítico de métodos mixtos y triangulación de datos que fueron organizados en categorías definidas para su análisis: 1) proceso de atención sanitaria, 2) información y conocimiento del estado de salud y situación clínica y 3) toma de decisiones.

\section{Participantes y escenarios}

Los participantes fueron:

1. Profesionales de la salud del Hospital Comarcal de Melilla de las unidades que a continuación se detallan, dado que no existe una específica de cuidados paliativos: Cirugía, (32 camas), Medicina Interna (32 camas) y Unidad de Cuidados Intensivos (UCl) (6 camas), seleccionados mediante muestreo intencional, manteniendo la proporcionalidad 1:2 médico/enfermera de acuerdo al número de médicos y enfermeras que componían la plantilla de las unidades en las que se realiza el estudio. Los criterios de inclusión fueron formar parte de la plantilla de las unidades mencionadas en el momento de recoger los 
datos y aceptar participar mediante firma de consentimiento informado. Cada participante constituyó un caso. Se excluyeron profesionales que llevasen trabajando en la unidad menos de seis meses, así como errores en la cumplimentación del cuestionario.

2. Historias clínicas de los pacientes fallecidos a lo largo de un año. Como criterios de inclusión, se estableció que la causa de exitus consignada en los registros de defunción del servicio de documentación fueran enfermedades crónicas como: Enfermedad Oncológica, Insuficiencia Renal Crónica (IRC), Insuficiencia Respiratoria/Enfermedad Pulmonar Obstructiva Crónica (EPOC), Insuficiencia Cardiaca (IC) e Insuficiencia Hepática (IH), excluyendo los fallecimientos acontecidos en las 24 horas posteriores al ingreso, así como las historias en las que se consignaron como causa de fallecimiento varios diagnósticos y aquellas en las que la enfermedad renal, respiratoria, cardíaca y/o hepática fueran la causa del exitus pero como complicaciones de la evolución de la enfermedad que padecieron. En total se analizaron 19 historias.

3. Familiar/Cuidador principal de pacientes fallecidos en el hospital con los siguientes criterios de inclusión: ser familiar de un paciente fallecido por enfermedad avanzada y/o terminal, implicado directamente en su cuidado durante el proceso de final de vida; haber transcurrido desde el fallecimiento entre 1 y 6 meses y aceptar participar en el estudio. Cada participante constituyó un caso. Se excluyeron familiares cuidadores que en el momento de realizar la encuesta mostraron dificultades para recordar datos, o se encontraban en un estado de abatimiento psicológico importante.

Tras aplicar los criterios de inclusión y exclusión, el estudio se realizó sobre una muestra de 63 sujetos, de los que 25 fueron profesionales, 19 familiares cuidadores y 19 historias de pacientes fallecidos.

El pequeño tamaño de la muestra está condicionado por la reducida capacidad de unidades y camas en las que se ubican los pacientes en situación terminal.

Una vez revisada la literatura y consultar grupos de expertos de diferentes disciplinas (enfermería, medicina, antropología, trabajo social y psicología), para la recogida de datos de profesionales y familiares, se confeccionaron cuestionarios ad hoc. El cuestionario para los profesionales estaba compuesto por 23 ítems mediante escala tipo Likert de cinco opciones, (1=totalmente en desacuerdo y $5=$ totalmente de acuerdo; $1=$ baja calidad y $5=a$ ta calidad; $1=$ poco común y $5=$ =muy común; $1=$ no es un obstáculo y $5=e$ un obstáculo grave, según la pregunta), dividido en 11 apartados que incluían datos sobre la estructura y proceso de atención; aspectos físicos, psicológicos, sociales, culturales y espirituales/existenciales de la atención prestada; aspectos relacionados con el cuidado del paciente moribundo; cuestiones éticas; la calificación de la propia práctica profesional, la de los colegas y la de la institución; la frecuencia de presentación de dilemas éticos en su contexto e identificación de obstáculos para el cuidado óptimo al final de la vida.

El cuestionario para conocer la opinión y percepción sobre la calidad de la atención en el proceso de final de vida los familiares, se diseñó con preguntas de respuesta dicotómica, organizadas en cinco epígrafes (datos socio-demográficos del familiar cuidador; datos referidos al familiar fallecido y su enfermedad; control de síntomas en 
el último mes; apoyo e información por parte de los profesionales y opinión sobre la atención en el proceso final de la vida). Los familiares participantes fueron contactados mediante llamada telefónica. Las entrevistas para implementar el cuestionario se llevaron a cabo en un único encuentro con cada informante, en su domicilio.

Tanto para los cuestionarios de familiares, como para el de profesionales, se obtuvo un consentimiento informado, firmado por los participantes.

Para el registro de datos de las Historias Clínicas, se elaboró un documento diseñado para facilitar la recogida de datos contrastables con los obtenidos del resto de fuentes, incluyendo variables sociodemográficas y clínicas, decisiones médicas de final de vida en los últimos 3 meses, tratamientos/intervenciones realizados en los últimos 14 días, 7 días y 48 horas, identificación de la situación clínica de terminalidad en los últimos 3 meses de vida y factores pronósticos relacionados con esta situación clínica. Para la selección e identificación se solicitaron datos de los registros de mortalidad durante un año, cuya causa básica de muerte incluyera alguna de las enfermedades mencionadas. Se accedió a las historias clínicas tras obtener el permiso del hospital y se revisaron los informes clínicos de ingreso y alta, evolución médica y evolución de enfermería.

\section{Análisis de datos}

Se utilizó el paquete estadístico SPSS (versión 20.0 Inc., Chicago, IL). Las variables cualitativas se describieron mediante frecuencias y las cuantitativas con la media y desviación estándar. Para la estadística inferencial se realizó el test de Chi cuadrado de Pearson. Se estableció una significación de $p=0.05$ para todos los análisis. Los resultados cuantitativos se organizaron agrupando los factores analizados en los cuestionarios según las categorías definidas, llevando a cabo una transformación de los datos e integrándolos en las categorías establecidas.

En cada categoría se comparó la información obtenida de cada fuente y se establecieron los elementos convergentes, complementarios y/o divergentes, así como si estos estaban presentes o ausentes.

\section{Consideraciones éticas}

La investigación contó con la aprobación del comité de ética e investigación del hospital. Todos los datos de carácter personal obtenidos en este estudio son confidenciales y han sido tratados conforme a la Ley Orgánica de Protección de Datos de Carácter Personal 15/99 y el Real Decreto 1720/2007, de 21 de diciembre, por el que se aprueba el Reglamento de desarrollo de esta ley.

\section{RESULTADOS}

La edad media de los familiares que participaron en el estudio fue de $57.2 \pm 14.49$ años, con una edad mínima de 29 años y máxima de 83. La edad media de los profesionales de la salud fue de $42.4 \pm 6.99$ años, con un mínimo de 28 años de edad y un máximo de 55; y la de los pacientes fallecidos consignados en las historias clínicas seleccionadas, fue de $76.5 \pm 9.66$ años, con un mínimo de 52 años de edad y un máximo de 92 . 
El resto de características sociodemográficas, así como los resultados referentes a la opinión de profesionales de la salud, cuidadores y datos registrados en las historias clínicas quedan recogidos en las Tablas 1, 2 y 3.

Tabla 1- Datos de los familiares y características del ser querido, Melilla, ME, España, 2019.

\begin{tabular}{|c|c|c|}
\hline \multicolumn{2}{|c|}{ Variables familiares } & \multirow{2}{*}{$\begin{array}{l}\begin{array}{l}N \\
(n=19)\end{array} \\
4 \\
15\end{array}$} \\
\hline Sexo & $\begin{array}{l}\text { Hombre } \\
\text { Mujer }\end{array}$ & \\
\hline Tipo de enfermedad & $\begin{array}{l}\text { Oncológicos } \\
\text { No oncológicos }\end{array}$ & $\begin{array}{l}12 \\
7\end{array}$ \\
\hline $\begin{array}{l}\text { Dónde paso más tiempo } \\
\text { el último mes }\end{array}$ & $\begin{array}{l}\text { Hospital } \\
\text { Domicilio }\end{array}$ & $\begin{array}{l}13 \\
6\end{array}$ \\
\hline Lugar de la muerte & $\begin{array}{l}\text { Hospital } \\
\text { Domicilio }\end{array}$ & $\begin{array}{l}19 \\
-\end{array}$ \\
\hline $\begin{array}{l}\text { Cómo considera que fue } \\
\text { la muerte de su familiar }\end{array}$ & $\begin{array}{l}\text { Buena } \\
\text { Regular } \\
\text { Mala } \\
\text { Muy mala }\end{array}$ & $\begin{array}{l}4 \\
3 \\
10 \\
2\end{array}$ \\
\hline
\end{tabular}

Tabla 2 - Datos generales de los profesionales y de la calidad asistencial en el fin de vida, Melilla, ME, España, 2019.

\begin{tabular}{|c|c|c|}
\hline \multicolumn{2}{|c|}{ Variables profesionales } & \multirow{2}{*}{$\begin{array}{l}\begin{array}{l}N \\
(n=25)\end{array} \\
99 \\
16\end{array}$} \\
\hline Sexo & $\begin{array}{l}\text { Hombre } \\
\text { Mujer }\end{array}$ & \\
\hline Profesional & $\begin{array}{l}\text { Facultativos } \\
\text { Enfermeros }\end{array}$ & $\begin{array}{l}9 \\
16\end{array}$ \\
\hline $\begin{array}{l}\text { Unidad / servicio de } \\
\text { trabajo }\end{array}$ & $\begin{array}{l}\text { Cirugía } \\
\text { UCl } \\
\text { Medicina Interna }\end{array}$ & $\begin{array}{l}8 \\
9 \\
8\end{array}$ \\
\hline $\begin{array}{l}\text { Cómo considera que es } \\
\text { la muerte de los } \\
\text { pacientes }\end{array}$ & $\begin{array}{l}\text { Muy buena } \\
\text { Buena } \\
\text { Regular } \\
\text { Mala } \\
\text { Muy mala }\end{array}$ & $\begin{array}{l}3 \\
6 \\
7 \\
3 \\
6\end{array}$ \\
\hline $\begin{array}{l}\text { Cómo considera que es } \\
\text { la calidad de su práctica } \\
\text { profesional con } \\
\text { pacientes al final de la } \\
\text { vida }\end{array}$ & $\begin{array}{l}\text { Alta } \\
\text { Buena } \\
\text { Aceptable } \\
\text { Regular } \\
\text { Baja }\end{array}$ & $\begin{array}{l}1 \\
16 \\
6 \\
2 \\
-\end{array}$ \\
\hline
\end{tabular}


Cómo considera que es Alta la calidad de la práctica profesional de sus colegas con pacientes al final de la vida

Aceptable 6

Regular 2

Baja 1

Tabla 3 - Datos generales de los pacientes, Melilla, ME, España, 2019.

\begin{tabular}{|c|c|c|}
\hline \multicolumn{2}{|c|}{ Historias clínicas pacientes } & $\begin{array}{c}N \\
(n=19)\end{array}$ \\
\hline Sexo & $\begin{array}{l}\text { Hombre } \\
\text { Mujer }\end{array}$ & $\begin{array}{c}11 \\
8\end{array}$ \\
\hline Enfermedad & $\begin{array}{l}\text { Oncológica } \\
\text { No oncológica }\end{array}$ & $\begin{array}{c}5 \\
14\end{array}$ \\
\hline $\begin{array}{l}\text { Diagnóstico } \\
\text { terminalidad }\end{array}$ & $\begin{array}{l}\text { Sí } \\
\text { No }\end{array}$ & $\begin{array}{c}6 \\
13\end{array}$ \\
\hline $\begin{array}{l}\text { Tiempo transcurrido } \\
\text { entre el diagnóstico y la } \\
\text { defunción }\end{array}$ & $\begin{array}{l}\text { Menos de } 48 \mathrm{~h} \\
\text { Una semana } \\
\text { Más de un mes }\end{array}$ & $\begin{array}{l}3 \\
2 \\
1\end{array}$ \\
\hline Vía central & $\begin{array}{l}\text { Sí } \\
\text { No }\end{array}$ & $\begin{array}{c}11 \\
8\end{array}$ \\
\hline Vía periférica & $\begin{array}{l}\text { Sí } \\
\text { No }\end{array}$ & $\begin{array}{c}13 \\
6\end{array}$ \\
\hline Sonda nasogástrica & $\begin{array}{l}\text { Sí } \\
\text { No }\end{array}$ & $\begin{array}{c}7 \\
12\end{array}$ \\
\hline Nutrición enteral & $\begin{array}{l}\text { Sí } \\
\text { No }\end{array}$ & $\begin{array}{c}7 \\
12\end{array}$ \\
\hline Nutrición parenteral & $\begin{array}{l}\text { Sí } \\
\text { No }\end{array}$ & $\begin{array}{c}4 \\
15\end{array}$ \\
\hline $\begin{array}{l}\text { Ventilación mecánica } \\
\text { Invasiva o no invasiva }\end{array}$ & $\begin{array}{l}\text { Sí } \\
\text { No }\end{array}$ & $\begin{array}{l}2 \\
17\end{array}$ \\
\hline Sonda vesical & $\begin{array}{l}\text { Sí } \\
\text { No }\end{array}$ & $\begin{array}{c}14 \\
5\end{array}$ \\
\hline Oxigenoterapia & $\begin{array}{l}\text { Sí } \\
\text { No }\end{array}$ & $\begin{array}{c}16 \\
3\end{array}$ \\
\hline Aerosoles & $\begin{array}{l}\text { Sí } \\
\text { No }\end{array}$ & $\begin{array}{c}9 \\
10\end{array}$ \\
\hline
\end{tabular}


En cuanto a las características del proceso de atención sanitaria, el análisis de los datos obtenidos mostró que, en general, los pacientes pasan su último mes de vida ingresados en el hospital, pero incluso cuando permanecen en el domicilio durante ese último mes, terminan falleciendo en el hospital. En las historias clínicas, los datos relativos al proceso de atención sanitaria, información/conocimiento y toma de decisiones eran muy escasos, y en pocas ocasiones se mostraban organizados y/o denominados de forma que pudieran ser identificados. Para evitar interpretaciones y suposiciones que pudieran entenderse como una manipulación de los resultados, se optó por constatar como ausentes los datos que no estuvieran identificados adecuadamente.

Proceso de atención sanitaria (Tabla 4).

Los componentes de esta categoría en los que se encontraron diferencias significativas entre familiares y profesionales se relacionaron con el control del dolor y de otros síntomas. Los profesionales consideraron de forma mayoritaria que el dolor y los síntomas se controlaron la mayor parte del tiempo mientras que para los familiares solo se consiguió a veces. Familiares y profesionales coincidieron en su valoración positiva respecto al acompañamiento del paciente durante el proceso y, en su valoración negativa respecto a la atención de necesidades espirituales. En las historias clínicas no se encontró ninguna información registrada relativa al proceso de atención sanitaria.

Tabla 4 - Proceso asistencial, Melilla, ME, España, 2019.

\begin{tabular}{|c|c|c|c|c|c|c|c|c|}
\hline \multicolumn{2}{|c|}{ Proceso asistencial } & \multicolumn{2}{|c|}{ Familiares } & \multicolumn{2}{|c|}{ Profesionales } & \multicolumn{2}{|c|}{$\begin{array}{l}\text { Historias } \\
\text { clínicas }\end{array}$} & \multirow{2}{*}{$\begin{array}{l}\text { Significación } \\
\text { Chi } \\
\text { cuadrado de } \\
\text { Pearson } \\
\left({ }^{*}\right)\end{array}$} \\
\hline \multirow{6}{*}{$\begin{array}{l}\text { Se controló } \\
\text { el dolor }\end{array}$} & & $\mathrm{n}$ & (\%) & $n$ & $(\%)$ & $n$ & (\%) & \\
\hline & $\begin{array}{l}\text { Siempre } \\
\text { La mayor }\end{array}$ & 1 & (5.26) & 7 & (28.0) & $\#$ & \# & \multirow{5}{*}{.000} \\
\hline & parte del & 3 & (15.78) & 11 & $(44.0)$ & $\#$ & \# & \\
\hline & $\begin{array}{l}\text { tiempo } \\
\text { A veces }\end{array}$ & 7 & (36.84) & 1 & $(4.0)$ & \# & \# & \\
\hline & $\begin{array}{l}\text { Pocas } \\
\text { veces }\end{array}$ & 5 & $(26.31)$ & 5 & $(20.0)$ & $\#$ & \# & \\
\hline & Nunca & 3 & (15.78) & 1 & $(4.0)$ & \# & \# & \\
\hline \multirow{5}{*}{$\begin{array}{l}\text { Se } \\
\text { controlaron } \\
\text { otros } \\
\text { síntomas }\end{array}$} & $\begin{array}{l}\text { Siempre } \\
\text { La mayor }\end{array}$ & 1 & $(5.26)$ & 7 & (28.0) & $\#$ & \# & \multirow{5}{*}{.000} \\
\hline & $\begin{array}{l}\text { parte del } \\
\text { tiempo }\end{array}$ & 4 & $(21,05)$ & 13 & $(52.0)$ & \# & \# & \\
\hline & A veces & 11 & $(57.89)$ & 1 & $(4.0)$ & $\#$ & \# & \\
\hline & $\begin{array}{l}\text { Pocas } \\
\text { veces }\end{array}$ & 2 & (10.52) & 3 & $(12.0)$ & $\#$ & $\#$ & \\
\hline & Nunca & 1 & $(5.26)$ & 1 & $(4.0)$ & \# & \# & \\
\hline
\end{tabular}




\begin{tabular}{|c|c|c|c|c|c|c|c|c|}
\hline \multirow{5}{*}{$\begin{array}{l}\text { Se da apoyo } \\
\text { a los } \\
\text { familiares }\end{array}$} & Siempre & 4 & $(22.2)$ & 4 & $(16.0)$ & \# & \# & \multirow{5}{*}{.000} \\
\hline & $\begin{array}{l}\text { La mayor } \\
\text { parte del }\end{array}$ & 10 & $(55.6)$ & 7 & $(28.0)$ & \# & \# & \\
\hline & A veces & 4 & (22.2) & 5 & $(20.0)$ & \# & \# & \\
\hline & $\begin{array}{l}\text { Pocas } \\
\text { veces }\end{array}$ & - & - & 4 & $(16.0)$ & \# & \# & \\
\hline & Nunca & - & - & 5 & $(20.0)$ & \# & \# & \\
\hline \multirow{2}{*}{$\begin{array}{l}\text { El paciente } \\
\text { tuvo } \\
\text { compañía }\end{array}$} & Si & 14 & (73.7) & 19 & $(76.0)$ & \# & \# & \multirow[b]{2}{*}{.000} \\
\hline & No & 5 & $(26.3)$ & 6 & $(24.0)$ & \# & \# & \\
\hline \multirow{2}{*}{$\begin{array}{l}\text { Se } \\
\text { atendieron } \\
\text { las } \\
\text { necesidades } \\
\text { espirituales }\end{array}$} & Sí & 8 & $(42.1)$ & 10 & $(40.0)$ & \# & \# & \multirow{2}{*}{.000} \\
\hline & No & 11 & (57.9) & 15 & $(60.0)$ & \# & \# & \\
\hline (\#) No se & recoge en la $\mathrm{Hi}$ & listor & $\begin{array}{l}\text { Clínica } \\
\text { amiliares }\end{array}$ & $\begin{array}{l}\text { Fam } \\
\text { Profes }\end{array}$ & $\begin{array}{l}\text { iliares/Profe } \\
\text { sionales }\end{array}$ & esiona & les/ $/ H^{\mathrm{a}} \mathrm{C}$ & ínicas $\left(^{* *}\right)$ \\
\hline \multicolumn{9}{|c|}{ Información y Conocimiento (Tabla 5) } \\
\hline \multicolumn{9}{|c|}{$\begin{array}{l}\text { Referente a la información y al conocimiento de que disponen la familia y el paciente, } \\
\text { se obtienen resultados muy dispares. El } 57,9 \% \text { de los familiares consideró que se les } \\
\text { informó sobre diagnóstico y pronóstico siempre o la mayor parte del tiempo. El } 40 \% \\
\text { de los profesionales opinan igual. Sin embargo, esta información se registra sólo en el } \\
31,6 \% \text { de las historias. }\end{array}$} \\
\hline \multicolumn{9}{|c|}{$\begin{array}{l}\text { Tabla } 5 \text { - Información y conocimientos de la familia / paciente, Melilla, ME, } \\
\text { España, } 2019 .\end{array}$} \\
\hline \multirow{2}{*}{\multicolumn{2}{|c|}{$\begin{array}{l}\text { Información y } \\
\text { conocimiento }\end{array}$}} & \multicolumn{2}{|c|}{ Familiares } & \multicolumn{2}{|c|}{ Profesionales } & \multicolumn{2}{|c|}{$\begin{array}{l}\text { Historias } \\
\text { clínicas }\end{array}$} & $\begin{array}{l}\text { Significación } \\
\text { Chi } \\
\text { cuadrado de } \\
\text { Pearson }\end{array}$ \\
\hline & & $\mathrm{n}$ & $(\%)$ & $n$ & $(\%)$ & $\mathrm{n}$ & $(\%)$ & $\left({ }^{*}\right)$ \\
\hline \multirow{5}{*}{$\begin{array}{l}\text { Se informó } \\
\text { diagnóstico y } \\
\text { pronóstico }\end{array}$} & $\begin{array}{l}\text { Siempre } \\
\text { La mayor }\end{array}$ & 4 & (21.1) & 6 & $(24.0)$ & 6 & (31.6) & \multirow{5}{*}{.000} \\
\hline & $\begin{array}{l}\text { parte del } \\
\text { tiempo }\end{array}$ & 7 & (36.8) & 4 & $(16.0)$ & - & - & \\
\hline & A veces & 6 & $(31.6)$ & 4 & $(16.0)$ & - & - & \\
\hline & $\begin{array}{l}\text { Pocas } \\
\text { veces }\end{array}$ & - & - & 5 & $(20.0)$ & - & - & \\
\hline & Nunca & 2 & (10.5) & 6 & $(24.0)$ & 13 & $(68.4)$ & \\
\hline \multirow{2}{*}{$\begin{array}{l}\text { Información } \\
\text { voluntades } \\
\text { anticipadas }\end{array}$} & $\mathrm{Si}$ & 5 & (26.3) & 3 & $(12.0)$ & \# & \# & \multirow{2}{*}{.000} \\
\hline & No & $\begin{array}{l}1 \\
4\end{array}$ & (73.7) & 22 & $(68.0)$ & \# & \# & \\
\hline
\end{tabular}




\begin{tabular}{|c|c|c|c|c|c|c|c|c|}
\hline \multirow{2}{*}{$\begin{array}{l}\text { Paciente } \\
\text { conocimiento } \\
\text { diagnóstico } \\
\text { terminalidad }\end{array}$} & $\mathrm{Si}$ & 6 & (31.6) & 16 & $(64.0)$ & 1 & (5.3) & \multirow[b]{2}{*}{.000} \\
\hline & No & $\begin{array}{l}1 \\
3\end{array}$ & (68.4) & 9 & $(36.0)$ & 18 & $(94.7)$ & \\
\hline \multirow{2}{*}{$\begin{array}{l}\text { Familiar } \\
\text { conocimiento } \\
\text { diagnóstico } \\
\text { terminalidad }\end{array}$} & $\mathrm{Si}$ & $\begin{array}{l}1 \\
5\end{array}$ & (78.9) & 16 & $(64.0)$ & 3 & $(15.8)$ & \multirow{2}{*}{.001} \\
\hline & No & 4 & (21.1) & 9 & $(36.0)$ & 16 & $(84.2)$ & \\
\hline
\end{tabular}

(\#) No se recoge en la Historia Clínica $\left(^{*}\right)$ Familiares/Profesionales $/ H^{a}$ Clínicas $\left(^{* *}\right)$

Familiares/Profesionales

Toma de decisiones.

Los datos comparados de las diferentes fuentes de información mostraron discrepancias importantes entre profesionales y familiares principalmente. Los familiares consideraron en más de la mitad de los casos (58.8\%) que se había tenido en cuenta su opinión y la del paciente, sin embargo, los profesionales dijeron en un $45.8 \%$ de los casos que no se contó con estas opiniones, al igual que las historias, en las que en el $89,5 \%$ no se registró esta opinión. Respecto a si el paciente tomó decisiones de forma directa, la respuesta fue negativa para todos los profesionales y para el $89.5 \%$ de los familiares. En las historias no existe ningún dato registrado sobre esto. En cuanto a decisiones sobre medidas concretas como la limitación del esfuerzo terapéutico (LET) y la indicación de No Reanimación Cardiopulmonar (RCP), la discrepancia de opiniones es significativa en el caso de la RCP. Para el $89.5 \%$ de los familiares no existió indicación de no reanimación, mientras que el $52 \%$ de los profesionales opinaron que sí se indicó. Los datos de las historias clínicas mostraron que sólo en un caso (5.3\%) se registró esta indicación. Sobre si el lugar de fallecimiento del paciente fue el de su preferencia, familiares y pacientes opinaron que no en un $89,5 \%$ y, el $68 \%$ de los profesionales que sí. En cuanto a la decisión de la sedación terminal, esta opción se dio según los familiares en un $16,7 \%$, frente al $32 \%$ de los profesionales que opinaron en el mismo sentido no quedando registro sobre esta decisión en las historias clínicas.

\section{DISCUSIÓN}

Los resultados muestran la escasez de datos relevantes recogidos en las historias clínicas; en concreto no se indica si el paciente tiene voluntades anticipadas o si ha manifestado sus preferencias y deseos de cómo le gustaría que fuese el proceso de fin de vida, lo que coincide con los resultados de otros estudios ${ }^{(18)}$. Entre las posibles explicaciones encontradas en la literatura revisada, hallamos la falta de competencias(19), conocimiento sobre requisitos legales y la falta de tiempo(20), información que resulta importante documentar en aras de garantizar la seguridad del paciente(21).

Otro hallazgo destacado, ha sido la constatación de la calidad de la atención sanitaria al final de la vida, que puede ser calificado como deficitario si nos atenemos a indicadores como el número de hospitalizaciones en los últimos treinta días de vida(6). Nuestros resultados muestran que todos los pacientes fallecieron en el hospital, y que prácticamente tres de cada cuatro estuvieron ingresados al menos durante el mes 
previo al fallecimiento o tuvieron hospitalizaciones repetidas, lo que parece relacionarse con la percepción de una mala experiencia. Sin embargo, los datos relacionados con la intensidad de las intervenciones, son indicativos de una buena calidad de la atención recibida.

En cuanto al control de síntomas, otros autores describen la atención prestada en los treinta días previos al fallecimiento y la percepción de los familiares, refleja la variabilidad en la satisfacción, en concreto con la disnea, así como una mayor insatisfacción con la atención general en el hospital con respecto al domicilio(22). Esta circunstancia no ha podido constatarse en nuestro estudio, ya que todos los pacientes fallecieron en el hospital.

Respecto a la información y conocimiento, las discrepancias se manifestaron en un sentido diferente. Los profesionales tuvieron una peor opinión respecto a la información ofrecida a los familiares, que los propios familiares. Esto puede explicarse porque quizás para los familiares es suficiente conocer la situación de gravedad sin entrar en detalles que podrían desestabilizar su equilibrio emocional. En este sentido, este resultado es coherente con la situación generalizada en nuestro país de conspiración de silencio y ocultación de la realidad solicitada por los familiares ${ }^{(7)}$.

Por último, en relación a la toma de decisiones, las discrepancias en los datos señalan un importante desacuerdo entre las percepciones de los profesionales y las de los familiares respecto de los principales temas objeto de decisión, y un deficitario registro de todo ello en las historias clínicas. Llama la atención que todos los profesionales afirmen que los pacientes no participan de forma directa en la toma de decisiones. Esta situación puede explicar otros datos destacables del desacuerdo, por ejemplo, en cuanto a la indicación de no reanimación, o sobre si el paciente murió donde quiso, o la decisión de administrar sedación terminal. Las discrepancias de los profesionales en la toma de decisiones, ha sido una cuestión detectada por otros investigadores. Además, el proceso de toma de decisiones y las preferencias del paciente y la familia, van a estar marcadas por creencias, valores y cultura. La influencia de estos aspectos, han de tenerse en cuenta como elementos decisivos en la comunicación y la vivencia del proceso fin de vida ${ }^{(18,23)}$.

El papel de enfermería es decisivo en la mejora de la percepción sobre la asistencia recibida. En otros países, las intervenciones de enfermería son altamente efectivas e incluyen no sólo el monitoreo de síntomas, también la coordinación de casos, educación en manejo de síntomas y el consejo terapéutico, llevándose a cabo principalmente en el domicilio o vía telemática. Estas intervenciones generalmente incluyen apoyo y formación de familiares o cuidadores principales ${ }^{(24)}$. Los resultados de este estudio nos indican que la intervención de la enfermera, tanto en la gestión de casos, como en la educación de manejo de síntomas y consejo terapéutico, es deficiente y puede mejorarse. Por ello, se debería incidir en aspectos como el apoyo psicológico, la formación e información a pacientes y familiares y ofrecer una intervención planificada que los incluya, como parte de los cuidados liderados por la enfermera.

Estos resultados deberían ser tenidos en cuenta en el diseño e implementación de planes de cuidados específicos que puedan, además, reducir los costes sanitarios.

Como limitaciones de este estudio podemos señalar que la generalización de los 
resultados sería prematura dado el tamaño de la muestra.

Dada la diversidad cultural de Melilla, consideramos importante, además, para futuras investigaciones, indagar en la satisfacción de las necesidades espirituales desde un enfoque intercultural puesto que otros estudios han mostrado que es un aspecto que atañe a la mayoría de pacientes ${ }^{(25)}$.

Sería interesante así mismo, estudiar cómo afectan determinados factores en la percepción sobre la calidad asistencial. Por ejemplo, el papel de enfermería, las necesidades espirituales del paciente/familiar $o$ el tipo de enfermedad terminal (oncológica o no). Y explorar el papel de los niveles asistenciales, ya que el manejo del cuidado paliativo muestra diferencias importantes entre atención primaria y especializada $^{(26)}$.

\section{CONCLUSIONES}

Este estudio proporciona una visión integrada sobre la situación de la atención sanitaria en el proceso de final de vida prestada en el Hospital de Melilla, mostrando áreas prioritarias de intervención, como la implicación del paciente en la toma de decisiones; cuestiones que deben abordarse para mejorar la atención en este proceso.

El contraste de la información obtenida de las historias clínicas, de los profesionales y los familiares muestra divergencias en cuanto a los aspectos fundamentales que contribuyen a mejorar la satisfacción durante el proceso de fin de vida.

Para mejorar la atención durante esta etapa parece necesario mejorar la formación específica de los profesionales e implementar la cumplimentación del registro documental, incluyendo el proceso informativo del diagnóstico y del pronóstico a pacientes y familiares.

Así mismo, es recomendable establecer procedimientos efectivos para favorecer la participación del paciente y la familia en la toma de decisiones.

\section{REFERENCIAS}

1. Esteban M, Grau, IC, Castells G, Bullicg I, Busquet X, Aranzana A; et al. Complejidad asistencial en la atención al final de la vida: criterios y niveles de intervención en atención comunitaria de salud. Med. paliat. 2015; 22(2): 69-80.

2. Florêncio MM, Lopes de Almeida M C. The illness experience: Palliative care given the impossibility of healing. Rev. Esc. Enferm. USP. 2016; 50:7-53.

3. Donato CT, Matuoka Y, Yamashita C, Salvetti MG. Effects of dignity therapy on terminally ill patients: a systematic review. Rev. Esc. Enferm. USP. 2016;50(6):10111021.

4. Fascioli A. Los cuidados paliativos al final de la vida: Expresión del reconocimiento del otro. Enfermería (Montev.) . 2016; 5(2):46-53.

5. Sepúlveda JM, Morales JM, Morales IM, Canca JC, Crespillo E, Timonet E. EI derecho a morir con dignidad en un hospital de agudos: un estudio cualitativo. Enferm. clín. (Ed. Impr.). 2014; 24(4): 211-218.

6. Lima JS, Asensio MR, Palomo JC Ramírez EB, Lima M. Razones para decidir morir 
en el hospital o en el domicilio. Visión de los/las profesionales. Gac. Sanit. (Barc., Ed. Impr.). 2018; 32(4): 362-368.

7. Lozano B, Huertas MM. Cuidados paliativos, cuidados compartidos. Cult. cuid. 2018; 49: 100-107.

8. Esteban M, Fernández J, Boira RM, Martínez T, Nadal S, Castell G. Concordancia entre la complejidad observada desde diferentes niveles asistenciales en pacientes crónicos complejos, con enfermedad avanzada o al final de la vida mediante un modelo de abordaje de la complejidad. Med. paliat. 2018; 4: 236-244.

9. García MT, Gil E, Fernández MC, Sanz EM, Dones M, Domínguez $A$, et al. Programa centralizado de formación en Cuidados Paliativos. Coordinación Regional de la Comunidad de Madrid 2010-2015. Educ. med. (Ed. Impr.). 2017; 18(2): 103-113.

10. Rodríguez I, Villamor A, Lahosa MT, Dorca M, Mas M, Salvador MI. Conocimientos y actitudes de enfermeras de onco-hematología ante el documento de voluntades anticipadas. Rev. cuba. enferm. 2018; 34(2): 1-15

11. Pascual MC. Información a familiares de pacientes en proceso final de vida en unidades de cuidados intensivos. Evaluación por parte de las enfermeras. Enferm. clín. (Ed. Impr.). 2014; 24(3):168-174.

12. Sánchez MR, Moreno M, Hueso C, Campos C, Varella A, Montoya R. Dificultades y factores favorables para la atención al final de la vida en residencias de ancianos: un estudio con grupos focales. Aten. prim. (Barc., Ed. Impr.). 2017; 49(5): 278-285.

13. Velarde JF, Luengo R, González R, González S, Álvarez B, Palacios D. Dificultades para ofrecer cuidados al final de la vida en las unidades de cuidados intensivos. La perspectiva de enfermería. Gac. Sanit. (Barc., Ed. Impr.). 2017; 31(4):299-304

14. Puerto HM. Calidad de vida en cuidadores familiares de personas en tratamiento contra el cáncer. Rev. cuid. (Bucaramenga. 2010). 2015; 6(2): 1029-1040.

15. Instituto Nacional de Estadística. Cifras oficiales de población resultantes de la revisión del Padrón municipal a 1 de enero [Internet]. INE; 2017 [citado 2018 mar. 1]. Disponible en: https://www.ine.es/jaxiT3/Datos.htm?t=2915

16. Guerrero RF, Prado M, Ojeda MG. Reflexión crítica epistemológica sobre métodos mixtos en investigación de enfermería. Enferm. univ. 2016; 13(4): 246-252.

17. Alzás T, García LM. La evolución del concepto de triangulación en la investigación social. Rev Pesqui Qualit. 2017; 5(8): 395-418.

18. Sepúlveda JM, Morales JM, Morales IM, Sánchez JC, Timonet EM, Rivas F. Análisis de la toma de decisiones compartidas al final de la vida en las historias clínicas. Med. paliat. 2017; 24(1):14-20.

19. Kreetapirom P, Jaruratanasirikul S, Pruphetkaew N. Audit of paediatric residents' medical records. Clin Teach. 2017; 14(3): 189-194.

20. López JJ, Moreno MG, Saavedra CH, Espinosa AL, Camacho JU. La importancia del registro clínico de enfermería: un acercamiento cualitativo. Nure Investigación. 2018; 15(93): 1-9.

21. Dy SM. Patient safety and end-of-life care: common issues, perspectives, and strategies for improving care. Am Hosp Care. 2016;33(8): 791-796.

22. Burge F, Lawson B, Asada Y, Mclntyre PF, Grufeld E, Flowerdew G. Bereaved family member perceptions of patient-focused family-centred care during the last 30 days of life using a mortality follow-back survey: does location matter?. BMC Palliat Care. 2014; $13: 25$

23. Manalo MF. End-of-Life Decisions about Withholding or Withdrawing Therapy: Medical, ethical, and Religio-cultural considerations. Palliat Care. 2013; 7: 1-5

24. Van Der Eerden M, Csikos A, Busa A, Hughes S, Radbruch L, Menten J, et al. Experiences of patients, family and professional caregivers with Integrated Palliative 
Care in Europe: protocol for an international, multicenter, prospective, mixed method study. BMC Palliat Care. 2014; 13:52. DOI: https://doi.org/10.1186/1472-684X-13-52 25. Singer AE, Goebel JR, Kim YS, Dy SM, Ahluwalia SC, Clifford M, et al. Populations and interventions for palliative and end-of-life care: a systematic review. J Palliat Med. 2016; 9(9): 995-1008.

26. Otte IC, Jung C. Bally K, Elger BS, Schildmann J. Interprofessional Silence at the End of Life: Do Swiss General Practitioners and Hospital Physicians Sufficiently Share Information About Their Patients?. J Palliat Med. 2016; 19(9): 983-986. 